\title{
Hyperbolically symmetric sources in Palatini $f(R)$ gravity
}

\author{
M. Z. Bhattia ${ }^{\mathrm{a}}$ Z. Yousaf ${ }^{\mathrm{b}}$, Z. Tariq $^{\mathrm{c}}$ \\ Department of Mathematics, University of the Punjab, Quaid-i-Azam Campus, Lahore 54590, Pakistan
}

Received: 14 October 2021 / Accepted: 31 October 2021 / Published online: 5 December 2021

(C) The Author(s) 2021

\begin{abstract}
A thorough examination of static hyperbolically symmetric matter configuration in the context of Palatini $f(R)$ gravitational theory has been carried out in this manuscript. Following the work of Herrera et al. (Phys. Rev. D 103: 024037, 2021) we worked out the modified gravitational equations and matching conditions using the Palatini technique of variation in Einstein-Hilbert action. It is found from the evaluations that the energy density along with the contribution of dark source terms is inevitably negative which is quite useful in explaining several quantum field effects, because negative energies are closely linked with the quantum field theory. Such negative energies may also assist in time-travel to the past and formation of artificial wormholes. Furthermore, we evaluated the algebraic expressions for the mass of interior hyperbolical geometry and total energy budget, i.e., the Tolman mass of the considered source. Also, the structure scalars are evaluated to analyze the properties of matter configuration. Few analytical techniques are also presented by considering several cases to exhibit the exact analytical static solutions of the modified gravitational equations.
\end{abstract}

\section{Introduction}

The dilemma of present-day accelerated expansion of the universe, i.e., the dark energy problem is an elementary issue in theoretical physics of modern history. Certain cosmological observations influenced by the survey of type-Ia Supernovae demonstrated that the cosmological models which require self-deceleration of the universe under its gravitational pull are un-natural. Few astrophysical scenarios have convincingly proven that at some time in the past, our universe went through a changeover to an expansion stage of de Sitter type.

\footnotetext{
${ }^{a}$ e-mail: mzaeem.math@pu.edu.pk (corresponding author)

b e-mail: zeeshan.math@pu.edu.pk

c e-mail: zohatariq24@yahoo.com
}

The elucidation of all such unanticipated observational outcomes needs a detailed knowledge about the cosmological evolution which is not possible without Einstein's invincible theory of gravitation, i.e., general theory of relativity (GR). Despite the fact that GR produces precise results at every inch in the space, there exist some intractable issues like inflation, speedy expansion of universe, dark matter and dark energy. These have been a matter of interest of many scientists and urged them to curiously look for the solutions. This laid the foundation for the discovery of extended gravitational theories (also termed as modified gravitational theories (MGT)) that were supposed to satisfactorily elucidate the dark energy origin, the interaction between dark matter and dark energy and the late-time expansionary phase of our universe, thus paving a path for the forthcoming research. Unfortunately, no extended theory has appropriately elucidated the abovementioned cosmological mysteries, however, they have provided new insights into the constitution and structure formation of our universe from its birth to current evolutionary stage and other intricate issues involving the dynamics of evolution. The $f(R)$ theory of gravity, being less sophisticated, straightforwardly generalizes GR by using $f(R)$ (a generic function of Ricci scalar $R$ ) instead of $R$ itself in the action principle. The Palatini version is taken into account, i.e., variation w.r.t. the metric tensor and the affinity (connection symbol) is deployed by considering them independent of each other.

The matter configuration inside a spacetime interrelates with its geometric curvature via field equations. To model a specific gravitational phenomena, the solutions to field equations are needed that can be acquired by imposing certain constraints e.g., symmetry assumptions, conformal flatness and other post-Newtonian approximations because of their high non-linearity. One out of such solutions is the renowned Schwarzschild solution for empty space and it is believed to be a unique static asymptotically flat solution to the field equations possessing spherical symmetry. From the word spherical symmetry, it is obvious that such a solution owns 
three killing vectors (mentioned in Eq. (2) in [1]), whereby invariance under rotations is depicted. In the form of polar coordinates, for $R>2 M$ i.e. outside the horizon, one may express Schwarzschild metric as

$$
\begin{aligned}
d s^{2}= & -\left(1-\frac{2 M}{R}\right) d t^{2}+\frac{1}{\left(1-\frac{2 M}{R}\right)} d r^{2} \\
& +r^{2}\left(d \theta^{2}+\operatorname{Sin}^{2} \theta d \phi^{2}\right),
\end{aligned}
$$

having the signature $(-,+,+,+)$. For $R<2 M$, the metric tensors $g_{t t}$ and $g_{r r}$ interchange their signs causing the signature to become $(+,-,+,+)$ which may depict a mathematical solution but physically it is insignificant. For $R=2 M$, an identifiable singularity emerges that can easily be eliminated by making use of coordinate transformation. A noticeable fact here is that the time-like killing vector outside the horizon turns into a space-like killing vector inside the horizon (implied by the fact that $g_{t t}$ and $g_{r r}$ interchange their signs). So, the coordinate transformation results in the loss of staticity inside the horizon. The staticity of the Schwarzschild solution over the whole spacetime is vital as it stems from the vacuum and sphericity conditions of the metric. So, in order to extend the solution from outside the horizon to its interior while maintaining the staticity everywhere, an absolutely different interpretation of horizon is requisite. Such an effort leads to a static solution that characterizes the spacetime as a combination of two absolute 4-manifolds: one representing the region exterior to the horizon and the other indicating the region inside the horizon. Both of these manifolds fabricate a single continuous topological manifold [2]. These $\theta-\phi$ submanifolds possess spherical symmetry in the region exterior to the horizon and hyperbolic symmetry inside the horizon and meet each other i.e. $R=2 M$ at a single curve $\theta=0$. Thus, for $R<2 M$, we acquire

$$
\begin{aligned}
d s^{2}= & -\left(\frac{2 M}{R}-1\right) d t^{2}+\frac{1}{\left(\frac{2 M}{R}-1\right)} d r^{2} \\
& +r^{2}\left(d \theta^{2}+\operatorname{Sinh}^{2} \theta d \phi^{2}\right),
\end{aligned}
$$

having the killing vectors as given by Eq. (4) in [1]. The motion of particles from exterior region of the horizon to its interior would be possible only when both of the manifolds intersect at $\theta=0$ which leads to the assumption of radial motion only. The plane as a whole is treated as a single mathematical manifold having a phase change at the point $R=2 \mathrm{M}$ because of signature change. Herrera et al. [1] propounded a comprehensive analysis on the motion of particles in the inner horizon of a blackhole with hyperbolic symmetry by investigating its geodesic equations in detail. The obtained outcomes are then compared with the previously obtained results for the case of spherically symmetrywhich revealed that a repulsive force in the horizon prevents the test particles from reaching the center. Herrera and Witten [2] put forward an alternative explanation of Schwarzschild black hole with a restriction that its solution must be static outside as well as inside the horizon. As an outcome of this restriction, they had to change the signature by implementing a transformation of one of the angle variables. The authors showed that a change of symmetry is producedwith a phase transition on the surface. Harrison [4] traced out the exact solutions of gravitational equations ofmotion by following themethod of separation of variables. Using these solutions along with the interchanging of variable technique, few other solutions are also obtained. All the solutions are then analyzed via geometrical and physical interpretations. Gaudin et al. [5] took into account a massless scalar field and investigated static solutions of Einstein's gravitational equations. Using some type of duality transformations, they developed a link of such solutions with the Kantowski-Sachs solutions. They also worked on the physical attributes of limiting case of a vacuum hyperbolic spacetime.

The Schwarzschild solution indicating the exterior region of an astronomical configuration is widely recognized as an appropriate gadget for analyzing the gravitational field outside a spherically symmetric mass. The region interior to the horizon had no appropriate mathematical formulation to explicate it [3]. In fact, it was propounded at first by Harrison [4] and later became the subject of interest of many researchers [5-10].

Description of gravitational phenomena in competition to GR has been the most discussed topic for the past many years. Nojiri and Odintsov [11] overviewed the gravitational alternatives to GR i.e. the modified theories by considering various kinds of models in $f(R), f(G)$ and $f(R, G)$ gravity. They showed that few such theories went successfully through the Solar system tests and have excellent cosmological structures i.e. these are capable of describing the latetime epoch naturally. They also mentioned the possibility to elucidate the coincidence issue that manifests the expansion of the cosmos. Meng and Wang [12] investigated the Palatini formalism to modify the gravity by utilizing an $\ln R$ term first introduced by Nojiri and Odintsov [13] in the action integral. They demonstrated that in Palatini formalism, the $\ln R$ term can explain the current exponential expansion and reduces to Friedmann evolution for the region having high red shift. They also discussed the issue associated with the extension of $\ln R$ gravity by the addition of $R^{m}$ terms. Bertolami et al. [14] derived equations of motion for highly massive particles in the context of $f(R)$ gravity theory. They demonstrated that an extra force is generated when an explicit coupling between lagrangian matter density and arbitrary function $f(R)$ is achieved. Such an extra force is found to be orthogonal to the four-velocity. In weak field limit, they also obtained the corresponding law of acceleration. Paul et al. [15] considered a polynomial function of the curvature $R$ known as the lagrangian density $f(R)$ in the Einstein-Hilbert action for vacuum. By taking the Robertson-Walker metric, 
highly non-linear field equations are obtained that are not adequate for analytic solution, so the authors adopted numerical technique to analyze the evolution of FRW universe. The models worked out by the authors may help in reproducing the current expansion phase of the universe. Lobo and Oliveira [16] constructed traversable wormholes in the background of $f(R)$ gravity theory. They imposed the condition that the matter in the wormhole fulfills the energy conditions which reveals that the higher order derivatives of the curvature terms violate the null energy condition depicting that the higher order terms support such wormhole geometries. Olmo et al. [17] provided an overview of the primary results such as the Tolman-Oppenheimer-Volkoff equation, mass-radius relations and Chandrasekhar's limit etc. in the background of modified theories of gravity. They combined the constraints and outcomes of both relativistic and non-relativistic stars and built a bridge between the observations and efforts of researchers working on variety of theories. Clifton et al. [18] presented a complete survey on the modified gravity theories along with their cosmological influences. They also reviewed the efforts to construct a Parameterized Post-Friedmannian technique useful to constrain the deviations from GR.

Solutions to Einstein's gravitational equations possessing hyperbolic symmetry have been an important topic among the researchers. Herrera et al. [19] considered dissipative fluid with hyperbolic symmetry and studied their general properties and found that the energy density comes out to be negative. The region in the center contains no element of the fluid so this region is described with the help of a vacuum cavity. By taking into account the quasi-homologous regime, few exact solutions are procured analytically.

Riemann tensor can be divided into certain parts by using its dual along with four velocity vector to attain few tensor quantities which in turn produce few scalar functions called the structure scalars. Such scalars appear to be connected with the fluid variables and play a crucial role in understanding the dynamics of astronomical object. Lanczos [20] found that the antidual part of Riemann tensor can be reduced to a contracted tensor quantity. From their analysis, the procured third order tensor having sixteen components and found that such a tensor can assist in integrating the field equations of a quadratic action by reducing them from fourth to second order. Herrera et al. [21] inspected static axially symmetric matter sources by evaluating certain dynamical equations e.g the field equations and the conservation equations. The inhomogeneity factors with the help of structure scalars are identified and few exact solutions are procured analytically, one out of which elucidates an incompressible spheroid with pressure isotropy. Bhatti et al. [22,23] constructed the modified structure scalars for spherically symmetric dissipative system by taking into account the Palatini $f(R)$ gravity theory from the point of view of a tilted observer with and without the presence of electromagnetic force. They evaluated certain dynamical equations including the Raychaudhuri equation and the Bianchi identities. With the help of theses dynamical equations, they analyzed the structure formation as well as the evolutionary stages of astronomical object. Bhatti and Tariq [24] used the technique of splitting the Riemann tensor into three parts and obtained few structure scalars in the context of GR in the presence of an electromagnetic force for a spherically symmetric dissipative fluid. The equations exhibit that the presence of electric charge results in an increase in the mass of spherical body.

A locally-defined set of vector fields that are linearly independent and orthonormal to each other are much more beneficial as compared to the coordinate basis. Such a set is termed as orthonormal tetrad and has been extensively employed in the literature. Recently, Bhatti et al. [25] pursued a comprehensive study on static hyperbolically symmetric sources by extending the work of Herrera et al. [26] to the case where the electromagnetic force is non-zero. Writing down the gravitational equations, they worked out few analytical solutions along with their corresponding generating functions. Tariq et al. [27] explored the pressure isotropy condition for a spherically symmetric dissipative astronomical system in the background of Palatini $f(R)$ gravity by working out a differential equation for Weyl scalar. They demonstrated that the factors like energy density, shear stress and dissipative flux are responsible for inducing the pressure anisotropy in the system. Herrera et al. [28] elucidated the changeover of an axially symmetric, radiating and dissipative fluid to its nonradiating state and evaluated the circumstances under which such a system reaches its equilibrium state. Herrera et al. [29] carried out a detailed investigation on the collapse of axially symmetric sources in GR by deploying all the basic equations. The equations represent a general dissipative fluid with pressure anisotropy. The techniques used by the authors have both analytical as well as numerical applications. Structure scalars are also identified and their role in the fluid dynamics is demonstrated. Herrera et al. [30] analyzed the exterior region of an axially symmetric source occupied by a null fluid produced as a result of dissipation process. The part played by the vorticity and its connection with the radiations is also determined.

This manuscript is assembled in the following pattern: Sect. 2 provides a bird's-eye view of the Palatini technique to arrive at the gravitational field equations. Further, it states the line element for hyperbolical symmetry along with the source, the orthonormal tetrad and the matching conditions. Section 3 incorporates the Palatini field equations, the hydrostatic equilibrium condition and the interior mass of the hyperbolic source. Section 4 comprises an expression for the intrinsic curvature tensor i.e. the Riemann tensor and an important relation between the Weyl scalar and the fluid variables. Section 5 includes an expression for the Tolman mass which is a measure of total energy budget of the source. Uti- 
lizing the breakdown technique of the Riemann tensor, Sect. 6 expresses certain modified scalar functions called the structure scalars. Section 7 demonstrates certain static solutions for the considered hyperbolic symmetry by taking different cases along with their sub-cases. Section 8 winds up the findings of the manuscript with a short summary and the values for Greek letters used in the dynamical equations representing dark source elements are provided in the Appendix.

\section{Modified action principle and hyperbolically symmetry source}

Modified gravitational theories come in handy when the gravitational field at large scales does not behave well in GR which is derived form the variation of standard HilbertEinstein action. In this scenario, it is found from the observations that a more general action is requisite to fully comprehend the gravitational interaction. One of the possible approach to attain such a generalized action is associated with the modification of geometric portion of the HilbertEinstein action. This modification aids in unraveling various cosmological mysteries including the dark matter problem.

The most convenient and unsophisticated modification of the geometric portion is the substitution of Ricci scalar $R$ with $f(R)$ (a generalized function of $R$ that contains higher order curvature invariants). Based on this generalized function, this theory is named as $f(R)$ gravitational theory and its principal constituent is that extra degrees of freedom can be accumulated into an effective form of stress-energy tensor which could generate dark source effects. Following is the expression for such a modified Hilbert-Einstein action

$S_{f(R)}=S_{G}+S_{M}=\frac{1}{2 \kappa} \int d^{4} x \sqrt{-g} f(R)+S_{M}$,

with $S_{M}$ nd $S_{G}$ signifying the matter lagrangian and gravitational part of action respectively To extract the modified field equations out of it, three different procedures exist in literature i.e. the metric approach, the Palatini approach and the metric-affine approach [31]. The Palatini approach relies on the fundamental idea that the connection $\Gamma_{\pi v}^{\alpha}$ is independent of the spacetime metric $g_{\pi v}$ i.e the geodesic structure of spacetime is dissociated from its metric structure. The implementation of such a variation leads us to the following modified field equations

$f^{\prime}(R) R_{\pi v}-\frac{1}{2} f(R) g_{\pi v}=\kappa T_{\pi v}$,

where $T_{\pi v}=-\frac{2}{\sqrt{-g}} \frac{\partial S_{M}}{\partial g_{\pi v}}$. Variation of Eq. (2) for independent Levi-Civita connection yields

$\nabla_{\pi}\left\{g^{\gamma \delta} \sqrt{-g} f_{R}(R)\right\}=0$.
A connection between the trace of stress-energy tensor $T$ and Ricci invariant $R$ is as follows

$R f_{R}(R)-2 f(R)=\kappa T$.

For vacuum, the Ricci invariant holds a constant value that can be defined using $f(R)$. So, we may use another metric tensor notation i.e. $h_{\pi v}=f_{R} g_{\pi v}$ from where cosmic attributes of vacuum space in the context of $f(R)$ theory can be observed [32]. Mathematically, the following relation may be established between this new form of metric tensor and the connection

$T_{\delta \zeta}^{\pi}=\frac{1}{2} h^{\pi \alpha}\left(h_{\alpha \zeta, \delta}+h_{\alpha \delta, \zeta}-h_{\delta \zeta, \alpha}\right)$.

Making use of Eqs. (3) and (4), we acquire

$$
\begin{aligned}
\frac{1}{f_{R}} & \left(\nabla_{\delta} \nabla_{\zeta} f_{R}-g_{\delta \zeta} \square f_{R}\right)-\frac{1}{2} g_{\delta \zeta}\left(R-\frac{f}{f_{R}}\right) \\
& +\frac{\kappa T_{\delta \zeta}}{f_{R}}-R_{\delta \zeta}+\frac{1}{2} g_{\delta \zeta} R \\
& +\frac{3}{2 f_{R}^{2}}\left[\frac{1}{2} g_{\delta \zeta}\left(\nabla f_{R}\right)^{2}-\nabla_{\delta} f_{R} \nabla_{\zeta} f_{R}\right]=0,
\end{aligned}
$$

with $\nabla_{\sigma}$ and $\square$ signifying the covariant derivative and the d'Alembert operator. Concisely, we can write this equation in the form of a single set of modified field equations as

$G_{\delta \zeta}=\frac{\kappa}{f_{R}}\left(T_{\delta \zeta}+\mathcal{T}_{\delta \zeta}\right) \equiv T_{\delta \zeta}^{e f f}$

The efforts to determine a matter configuration sourcing the metric defined in Eq. (1) revealed that this metric basically indicates the interior region of the horizon which, in accordance with the observations, can possess hyperbolic symmetry only. We analyze such a hyperbolic distribution of matter that may or may not be bounded by the surface $\Sigma^{e}$ from the exterior but needfully bounded from the interior by the surface $\Sigma^{i}$. The line element is as follows

$d s^{2}=e^{v} d t^{2}-e^{\lambda} d r^{2}-r^{2} d \theta^{2}-r^{2} \sinh ^{2} \theta d \phi^{2}$,

with spacetime variables $v$ and $\lambda$ dependent on the radial coordinate only. The configuration of matter and energy indicated by the two-rank symmetric tensor $T_{\delta \zeta}$ is given as

$T_{\delta \zeta}=(\mu+P) V_{\delta} V_{\zeta}-P g_{\delta \zeta}+\Pi_{\delta \zeta}$,

with $\mu$ and $\Pi_{\delta \zeta}$ indicating the energy density and the anisotropic pressure respectively and $V_{\delta}$ is the fluid fourvelocity defined in covariant notation as $\left(e^{\nu / 2}, 0,0,0\right)$.

If orthonormal bases exist at some point, it provides an aid in specifying a locally inertial frame to express the components of tensors as observed by an observer at rest in 
that frame. The observer then possess a set of space axes (orthonormal) provided by spatial vectors $\mathbf{e}_{\eta}$. The combination of spatial axes with the observer's 4-velocity yields orthonormal tetrad. This observer's tetrad assists in splitting various tensors into their space-like and time-like parts and in gravitational theories, it helps in drawing out physical quantities from geometric objects. Following [26], the canonical orthonormal tetrad for our case is defined as

$$
\begin{aligned}
e_{\beta}^{(0)}=V_{\beta} ; & e_{\beta}^{(1)}=K_{\beta}=\left(0,-e^{\lambda / 2}, 0,0\right) ; \\
e_{\beta}^{(2)} & =L_{\beta}=(0,0,-r, 0) \\
e_{\beta}^{(3)} & =S_{\beta}=(0,0,0,-r \sinh \theta)
\end{aligned}
$$

which (after some manipulations provided in [26]) transforms the stress-energy tensor as

$$
\begin{aligned}
T_{\delta \zeta}= & \left(\mu+P_{z z}\right) V_{\delta} V_{\zeta}-P_{z z} g_{\delta \zeta} \\
& +\left(P_{x x}-P_{z z}\right) K_{\delta} K_{\zeta} .
\end{aligned}
$$

Here, $P_{x x}, P_{x y}, P_{y x}, P_{y y}$ and $P_{z z}$ illustrate the value of stresses in the directions indicated by their subscripts. Furthermore, the anisotropic tensor takes the below-mentioned form

$\Pi_{\delta \zeta}=\Pi\left(K_{\delta} K_{\zeta}+\frac{h_{\delta \zeta}}{3}\right)$,

where $P_{x x}-P_{z z}=\Pi$. The isotropic stress has the form $P=\frac{P_{x x}}{3}+\frac{2 P_{z z}}{3}$.

Since the interior region becomes a hyperboloid of two sheets [2], it is evident that the matter particles have no access to the region at the center of symmetry. Thus, a vacuum cavity emerges around the central region which demonstrates that the interior region becomes bounded too. The surface that bounds the matter from outside the horizon is represented as $\Sigma^{e}$ having equation $r=r_{\Sigma^{e}}=$ constant whereas the surface that bounds the matter from inside is represented as $\Sigma^{i}$ having equation $r=r_{\Sigma^{i}}=$ constant. The junction conditions for both the surfaces imply

$e^{\nu_{\Sigma^{e}}}=\frac{2 M}{r_{\Sigma^{e}}}-1, \quad e^{\lambda_{\Sigma^{e}}}=\frac{1}{\frac{2 M}{r_{\Sigma^{e}}}-1}, \quad P_{x x}^{(e f f)}\left(r_{\Sigma^{e}}\right)=0$,

$e^{\nu_{\Sigma^{i}}}=1, \quad e^{\lambda \Sigma^{i}}=1, \quad P_{x x}^{(e f f)}\left(r_{\Sigma^{i}}\right)=0, \quad m\left(r_{\Sigma^{i}}\right)=0$.

\section{Modified gravitational equations}

Palatini $f(R)$ equations acquired by substituting the nonzero components of tensors in Eq. (5) take the following form

$$
\begin{aligned}
8 \pi \mu & =f_{R}\left(-\frac{1+e^{-\lambda}}{r^{2}}+\frac{\lambda^{\prime} e^{-\lambda}}{r}\right)+\xi_{1} \\
8 \pi P_{r} & =f_{R}\left(\frac{1+e^{-\lambda}}{r^{2}}+\frac{v^{\prime} e^{-\lambda}}{r}\right)+\xi_{2}, \\
8 \pi P_{\perp} & =f_{R}\left\{\frac{e^{-\lambda}}{2}\left(v^{\prime \prime}-\frac{\lambda^{\prime} v^{\prime}}{2}+\frac{v^{\prime 2}}{2}-\frac{\lambda^{\prime}}{r}+\frac{v^{\prime}}{r}\right)\right\}+\xi_{3} .
\end{aligned}
$$

The conservation equation law i.e. $T_{\beta ; \delta}^{\delta}=0$ generates the hydrostatic equilibrium equation as follows

$\frac{d P_{r}}{d r}+\frac{1}{2}\left(\frac{d v}{d r}\right)\left(\mu+P_{r}\right)+\frac{2 \Pi}{r}+\tilde{\chi}_{(D)}=0$.

The expression for Misner-sharp mass function $m=m(r)$ for the interior region of the horizon is

$m(r)=\frac{r}{2}\left(e^{-\lambda}+1\right)$.

Using this value in the field equation associated with the energy density i.e. Eq. (9), we can relate the energy density with the geometric mass within the horizon as

$m(r)=-4 \pi \int_{0}^{r}\left(\frac{\mu r^{2}}{f_{R}}-\frac{\xi_{1} r^{2}}{8 \pi f_{R}}\right) d r$.

This expression depicts that geometric mass is negative which is unphysical because it is absolute i.e. it can never admit negative values. To avoid such a conflict, we have to assume negative energy density which is physically possible (dark energy is an example). So, we replace $-\mu$ by $|\mu|$ which yields the following outcome

$m(r)=4 \pi \int_{r_{\text {min }}}^{r}\left(\frac{|\mu| r^{2}}{f_{R}}+\frac{\xi_{1} r^{2}}{8 \pi f_{R}}\right) d r$.

The radial derivative of the spacetime variable $v$ turns out to be equal to

$\frac{d \nu}{d r}=2\left\{\frac{r^{3}\left(8 \pi P_{r}-\xi_{2}\right)-2 m f_{R}}{r f_{R}(2 m-r)}\right\}$.

Equation (12) on substituting the value of $d v / d r$ becomes

$$
\begin{aligned}
& \frac{d P_{r}}{d r}+\left(P_{r}-|\mu|\right)\left\{\frac{r^{3}\left(8 \pi P_{r}-\xi_{2}\right)-2 m f_{R}}{r f_{R}(2 m-r)}\right\} \\
& +\frac{2 \Pi}{r}+\tilde{\chi}_{(D)}=0 .
\end{aligned}
$$

Physically, this expression depicts that in the context of Palatini $f(R)$ gravity, the pressure gradient, the passive gravitational mass density $\left(P_{r}-|\mu|\right)$ along with the active gravitational mass, the pressure anisotropy and the dark source 
constituents must cancel the effect of each other to make the conservation of mass and energy possible.

\section{The intrinsic curvature tensor}

A symmetric trace-free tensor quantity of rank two defined by $E_{\delta \zeta}=C_{\delta \gamma \zeta \pi} V^{\gamma} V^{\pi}$ is termed as an electric part of Weyl tensor. It aids in the comprehension of tidal forces acting on an astronomical object in the manifold. Using the orthonormal tetrad, we may express it as $E_{\delta \zeta}=\epsilon\left(K_{\delta} K_{\zeta}+h_{\delta \zeta} / 3\right)$ with $\epsilon$ being equal to

$$
\begin{aligned}
\epsilon= & -\frac{v^{\prime \prime} e^{-\lambda}}{4}-\frac{v^{\prime 2} e^{-\lambda}}{8}+\frac{\lambda^{\prime} v^{\prime} e^{-\lambda}}{8} \\
& +\frac{v^{\prime} e^{-\lambda}}{4 r}-\frac{\lambda^{\prime} e^{-\lambda}}{4 r}-\frac{e^{-\lambda}}{2 r^{2}}-\frac{1}{2 r^{2}} .
\end{aligned}
$$

From the modified gravitational equational equations (9)(11) and Eqs. (13) and (18), one may acquire

$$
\frac{3 m}{r^{3}}=\frac{1}{f_{R}}\left[4 \pi|\mu|+4 \pi \Pi+\frac{\xi_{1}-\xi_{2}-\xi_{3}}{2}\right]-\epsilon
$$

Evaluating the radial derivative of this expression along with the usage of Eq. (14), we attain

$$
\begin{aligned}
\epsilon= & \frac{4 \pi \Pi}{f_{R}}+\frac{\xi_{1}-\xi_{2}-\xi_{3}}{2} \\
& +\frac{4 \pi}{r^{3}} \int_{0}^{r}\left\{\left(\frac{|\mu|}{f_{R}}\right)^{\prime} \tilde{r}^{3}-\frac{3 \xi_{1} \tilde{r}^{2}}{2 f_{R}}\right\} d \tilde{r} .
\end{aligned}
$$

Evidently, the dark source elements have a direct relation with $\epsilon$ which depicts that such elements give rise to the gravitational pull of the astronomical object which, in turn, results in the greater tidal force generated by it. For the mass function, we find

$$
m(r)=\frac{4 \pi|\mu| r^{3}}{3 f_{R}}-\frac{4 \pi}{3} \int_{0}^{r}\left\{\left(\frac{|\mu|}{f_{R}}\right)^{\prime} \tilde{r}^{3}-\frac{3 \xi_{1} \tilde{r}^{2}}{2 f_{R}}\right\} d \tilde{r}
$$

When compared with Eqs. (43) and (44) of [26], it is evident that the curvature amendment causes an increase in the tidal force as well as the geometric mass of the object under consideration. For a specific $f(R)$ model, one may be able to describe the extent to which the increment is produced e.g. for the Starobinsky model $f(R)=R+\alpha R^{2}$ where the curvature amendment is quadratic in $R$, the dark source elements would involve curvature invariants up to second order for the case of Palatini $f(R)$ and fourth order for the case of metric $f(R)$ gravity.

\section{Total energy budget of the source}

The mass function describing the total mass and energy content of the source is called as the Tolman mass i.e. the active gravitational mass. Its mathematical expression mentioned in Eq. (45) of [26] produces the following result for the case of hyperbolic symmetry in the background of Palatini $f(R)$ gravitational theory.

$$
\begin{aligned}
& m_{T}=\int_{0}^{2 \pi} \int_{0}^{\pi} \int_{0}^{r} \sqrt{-g}\left(T_{0}^{0}(e f f)\right. \\
& \left.-T_{1}^{1^{(e f f)}}-2 T_{2}^{2(e f f)}\right) d \tilde{r} d \theta d \phi .
\end{aligned}
$$

With the aid of Palatini field equations, we find

$$
\begin{aligned}
m_{T}= & 2 \pi(\cosh \pi-1) \int_{0}^{r} \frac{e^{\frac{v+\lambda}{2}} \tilde{r}^{2}}{f_{R}} \\
& \times\left(-|\mu|+P_{r}+2 P_{\perp}+\frac{\xi_{1}+\xi_{2}+2 \xi_{3}}{8 \pi}\right) d \tilde{r},
\end{aligned}
$$

which can be integrated to acquire an interesting relation connecting the Tolman mass with the spacetime variable $v$ in the presence of dark source elements as follows

$m_{T}=\frac{\cosh \pi-1}{4}\left\{v^{\prime} e^{\frac{v-\lambda}{2}} r^{2}+\tau_{(D)}\right\}$.

Using Eq. (16),

$m_{T}=\frac{\cosh \pi-1}{4}\left[e^{\frac{v+\lambda}{2}}\left(\frac{r^{3}\left(8 \pi P_{r}-\xi_{2}\right)-2 m f_{R}}{f_{R}}\right)+\tau_{(D)}\right]$,

with

$\tau_{(D)}=\int_{0}^{r} \frac{\tilde{r}^{2} e^{\frac{v+\lambda}{2}}}{f_{R}}\left(\frac{\xi_{1}+\xi_{2}+2 \xi_{3}}{4 \pi}\right) d \tilde{r}$.

From Eq. (14), it is evident that we avoided the negativity of Misner-sharp mass by replacing $-\mu$ with $|\mu|$. In the same manner, $4 \pi r^{3} P_{R}-\frac{\xi_{2}}{2}$ must be less than $m f_{R}$ to obtain the negative value for Tolman mass function which would indicate the repulsive nature of gravitational field around the considered astronomical object.

To comprehend the kinematics of fluid particles, let us evaluate the magnitude of 4-acceleration of the fluid configuration defined by $a_{\delta}=V_{\delta ; \beta} V^{\beta}$. In the context of Palatini $f(R)$ gravity, it turns out to be equal to

$a=e^{-\lambda / 2}\left\{\frac{v^{\prime 2}}{4}+\frac{f_{R}^{\prime 2}}{4 f_{R}^{2}}+\frac{v^{\prime} f_{R}^{\prime}}{2 f_{R}}\right\}^{1 / 2}$ 
Relating it with the Tolman mass, we can write

$a^{2}=\left[\frac{2 m_{T} e^{\frac{-\nu}{2}}}{r^{2}(\cosh \pi-1)}-\frac{\tau_{(D)} e^{-\nu / 2}}{r^{2}}\right]^{2}+\frac{f_{R}^{\prime 2} e^{-\lambda}}{4 f_{R}^{2}}+\frac{\nu^{\prime} f_{R}^{\prime} e^{-\lambda}}{2 f_{R}}$,

which points out that if $4 \pi r^{3} P_{R}-\frac{\xi_{2}}{2}<m f_{R}$, the acceleration of the fluid particles act radially inward. This provides additional support to the fact that gravitational force in our case has repulsive nature. Employing the $r$-derivative of Eq. (23) and using Eq. (25), we find

$$
\begin{aligned}
m_{T}^{\prime}-\frac{3 m_{T}}{r}= & -\left(\frac{\cosh \pi-1}{2}\right) r^{2} e^{\frac{v+\lambda}{2}} \\
& \times\left[\epsilon+\frac{4 \pi \Pi}{f_{R}}-\frac{\xi_{1}+\xi_{3}}{f_{R}}+\frac{\tau_{(D)} e^{\frac{-v-\lambda}{2}}}{2 r^{2}}\right] .
\end{aligned}
$$

Completing the derivative on the L.H.S by multiplying by $r^{4}$ and integrating w.r.t $r$, we get

$$
\begin{aligned}
m_{T}= & \left(m_{T}\right)_{\Sigma_{e}}\left(\frac{r^{3}}{r_{\Sigma^{e}}^{3}}\right)+\left(\frac{\cosh \pi-1}{2}\right) r^{3} \int_{r}^{r_{\Sigma^{e}}} \frac{e^{\frac{v+\lambda}{2}}}{\tilde{r}} \\
& \times\left[\epsilon+\frac{4 \pi \Pi}{f_{R}}-\frac{\xi_{1}+\xi_{3}}{f_{R}}+\frac{\tau_{(D) e^{\frac{-v-\lambda}{2}}}^{2}}{2 r^{2}}\right] d \tilde{r} .
\end{aligned}
$$

To relate the Tolman mass with the fluid variables i.e. the energy density inhomogeneity and pressure anisotropy, we substitute the value of $\epsilon$ in the above expression and find

$$
\begin{aligned}
m_{T}= & \left(m_{T}\right)_{\Sigma^{e}}\left(\frac{r^{3}}{r_{\Sigma^{e}}^{3}}\right)+\left(\frac{\cosh \pi-1}{2}\right) r^{3} \int_{r}^{r_{\Sigma^{e}}} \frac{e^{\frac{v+\lambda}{2}}}{\tilde{r}} \\
& \times\left\{\frac{4 \pi}{r^{3}}\left(\frac{|\mu|}{f_{R}}\right)^{\prime} s^{3}-\frac{3 \xi_{1} s^{2}}{2}\right\} d s \\
& +\frac{8 \pi \Pi}{f_{R}}-\frac{\tau_{(D)} e^{\frac{-v-\lambda}{2}}}{2 \tilde{r}^{2}}-\frac{\xi_{1}+\xi_{2}+3 \xi_{3}}{2 f_{R}} .
\end{aligned}
$$

In comparison with Eq. (54) of [26], the above expression depicts that the dark source elements also affect the total mass and energy distribution of the system. Just like the geometric mass, the Tolman mass is also affected by including higher order curvature invariants in the theory.

\section{Modified structure scalars}

The intrinsic curvature tensor i.e., the Riemann tensor has four indices. When acted upon by the Levi-Civita tensor, it yields its dual which can further be contracted with a unit time-like vector (4-velocity in our case) to obtain a new tensor quantity having two indices. Such formalism results in the resolution of Riemann tensor into its electric and magnetic parts which have great significance as they lead to certain scalar functions called the Structure Scalars. The mathematical procedure is well-defined by Herrera et al. in [33] and utilized by Bhatti et al. in [22] for the case of Palatini $f(R)$ gravity. So, we have

$$
\begin{aligned}
Y_{\delta \zeta} & =E_{\delta \zeta}+4 \pi \Pi+\frac{4 \pi}{3}(\mu+3 P) h_{\delta \zeta}+\chi_{\delta \zeta}, \\
X_{\delta \zeta} & =-\frac{8 \pi|\mu|}{3} h_{\delta \zeta}+4 \pi \Pi_{\delta \zeta}-E_{\alpha \xi}+\psi_{\delta \zeta}, \\
Z_{\delta \zeta} & =0
\end{aligned}
$$

The tensors in Eqs. (27) and (28) can further be decomposed into trace and trace-free parts as follows

$$
\begin{aligned}
X_{T} & =-8 \pi|\mu|+\bar{\psi}_{(D)}, \\
X_{T F} & =4 \pi \Pi-\varepsilon+\psi_{(D)}^{*},
\end{aligned}
$$

and

$$
\begin{aligned}
Y_{T} & =4 \pi(-|\mu|+3 P)+\bar{\chi}_{(D)}, \\
Y_{T F} & =4 \pi \Pi-\varepsilon+\chi_{(D)}^{*},
\end{aligned}
$$

where

$$
\psi_{(D)}^{*}=\frac{\psi_{\delta \zeta}}{K_{\delta} K_{\zeta}+\frac{h_{\delta \zeta}}{3}}, \quad \chi_{(D)}^{*}=\frac{\chi_{\delta \zeta}}{K_{\delta} K_{\zeta}+\frac{h_{\delta \zeta}}{3}} .
$$

It is clearly visible that each scalar function is associated with some characteristics of fluid configuration, for instance, the structure scalar $X_{T}$ is associated with the description of energy density of the fluid in addition to the dark source elements. Using Eq. (20), the trace-free scalar $Y_{T F}$, comes out to be directly related with the energy density inhomogeneities and dark source elements as follows

$$
\begin{aligned}
X_{T F}= & -\frac{4 \pi}{r^{3}} \int_{0}^{r}\left\{\left(\frac{|u|}{f_{R}}\right)^{\prime} \tilde{r}^{3}-\frac{3 \xi_{1} \tilde{r}^{2}}{2 f_{R}}\right\} d \tilde{r} \\
& -\frac{\xi_{1}-\xi_{2}-\xi_{3}}{2 f_{R}}+\psi_{(D)}^{*} .
\end{aligned}
$$

Similarly, for $Y_{T F}$, we find

$$
\begin{aligned}
Y_{T F}= & \frac{8 \pi \Pi}{f_{R}}+\frac{4 \pi}{r^{3}} \int_{0}^{r}\left\{\left(\frac{|u|}{f_{R}}\right)^{\prime} \tilde{r}^{3}-\frac{3 \xi_{1} \tilde{r}^{2}}{2 f_{R}}\right\} d \tilde{r} \\
& +\frac{\xi_{1}-\xi_{2}-\xi_{3}}{2 f_{R}}+\chi_{(D)}^{*} .
\end{aligned}
$$

Furthermore, the combination of trace-free scalar functions $X_{T F}$ and $Y_{T F}$ is found to be associated with the local stress anisotropy with the addition of dark source elements i.e.

$X_{T F}+Y_{T F}=\frac{8 \pi \Pi}{f_{R}}+\left(\psi_{(D)}^{*}+\chi_{(D)}^{*}\right)$.

On comparing Eqs. (30)-(36) with Eqs. (69)-(74) in [26], it becomes evident that the curvature amendments affect the 
structure scalars too. To explore the scalars $Y_{T}$ and $Y_{T F}$, we get back to the expressions for Tolman mass in Eqs. (22) and (26). Using Eqs. (32) and (36), we find

$$
\begin{aligned}
m_{T}= & \left(m_{T}\right)_{\Sigma e}\left(\frac{r}{r_{\Sigma e}}\right)^{3}+\left(\frac{\cosh \pi-1}{2}\right) r^{3} \int_{r}^{r_{\Sigma e}} \frac{e^{\frac{v+\lambda}{2}}}{\tilde{r}} \\
& \times\left(Y_{T F}-\frac{\tau_{(D)} e^{\frac{-v-\lambda}{2}}}{2 \tilde{r}^{2}}-\frac{\xi_{1}+\xi_{2}+3 \xi_{3}}{f_{R}}-\chi_{(D)}^{*}\right) d \tilde{r}, \\
m_{T}= & \frac{\cosh \pi-1}{2} \int_{0}^{r} \frac{\tilde{r}^{2} e^{(v+\lambda) / 2}}{f_{R}} \\
& \times\left(Y_{T}-\bar{\chi}_{(D)}+\frac{\xi_{1}+\xi_{2}+2 \xi_{3}}{2}\right) d \tilde{r} .
\end{aligned}
$$

Evidently, the total mass inside the fluid (bounded between the radius $r$ and the surface $r_{\Sigma^{e}}$ ) can be expressed by using the structure scalar $Y_{T F}$. This scalar explains all the basic characteristics of the fluid configuration i.e the energy density inhomogeneity and the anisotropic stress that generate complexity in a system. For this reason, it can be termed as the complexity factor. $Y_{T}$ is directly linked with the Tolman mass density.

\section{Few static solutions}

This section copes with the issue of determining all the possible static hyperbolically symmetric solutions i.e. the values for the spacetime variables in the context of Palatini $f(R)$ gravitational approach. Using the format of [26], we first develop a general formalism and then implement certain constraints to acquire the possible solutions to the modified gravitational equations. From Eqs. (10) and (11),

$$
\begin{aligned}
8 \pi\left(P_{r}-P_{\perp}\right)= & f_{R}\left(\frac{1+e^{-\lambda}}{r^{2}}\right)-\frac{e^{-\lambda} f_{R}}{2} \\
& \times\left(v^{\prime \prime}+\frac{v^{\prime 2}}{2}-\frac{\lambda^{\prime} v^{\prime}}{2}-\frac{v^{\prime}}{r}-\frac{\lambda^{\prime}}{r}\right)+\xi_{2}-\xi_{3},
\end{aligned}
$$

which on using new variables $e^{-\lambda}=y, z=v^{\prime} / 2+1 / r$ becomes

$$
\begin{aligned}
y^{\prime} & +y\left[\frac{4}{r^{2} z}+2 z+\frac{2 z^{\prime}}{z}-\frac{6}{r}\right] \\
= & \frac{2}{z}\left[\frac{1}{r^{2}}-\frac{8 \pi \Pi}{f_{R}}+\frac{\xi_{2}-\xi_{3}}{f_{R}}\right] .
\end{aligned}
$$

Solving this first order differential equation and putting back the value of $y$, we find

$$
e^{\lambda(r)}=\frac{z^{2} e^{\int\left(2 z+\frac{4}{r^{2}}\right) d r}}{r^{6}\left[2 \int\left\{\frac{z}{r^{8}}\left(\frac{8 \pi \Pi r^{2}}{f_{R}}+1+\frac{\xi_{2}-\xi_{3}}{f_{R}}\right) e^{\int\left(2 z+\frac{4}{z r^{2}}\right) d r}\right\} d r+A\right]},
$$

where $A$ denotes the integration constant. This formalism reveals that all the possible solutions can be well-described with the aid of two generating functions $z$ and $\Pi$. So, for the fluid variables, we acquire

$$
\begin{aligned}
4 \pi|\mu|= & \frac{f_{R}}{r^{2}}\left(m^{\prime}-\frac{\xi_{1} r^{2}}{2 f_{R}}\right), \\
4 \pi P_{r}= & \frac{2 f_{R}[z r(2 m-r)-2 m+r]+\xi_{2} r^{3}+2 m f_{R}}{2 r^{3}}, \\
8 \pi P_{\perp}= & f_{R}\left(\frac{2 m}{r}-1\right)\left\{z^{\prime}+z^{2}-\frac{z}{r}+\frac{1}{r^{2}}\right\} \\
& +z\left(\frac{m^{\prime}}{r}-\frac{m}{r^{2}}\right)+\xi_{3} .
\end{aligned}
$$

Now, we proceed towards the determination of certain explicit solutions to modified gravitational equations along with their generating functions.

\subsection{Conformally flat constraint}

The simplest constraint that may allow a static solution to Eqs. (9)-(11) is the conformally flat constraint. It is based on the hypothesis that the Weyl scalar vanishes i.e. $\epsilon=0$. This constraint when used in Eq. (18) allows the completion of derivatives which in turn produces the following result

$$
\frac{\partial}{\partial r}\left\{\frac{v^{\prime} e^{-\lambda}}{2 r}\right\}+e^{-\nu-\lambda} \frac{\partial}{\partial r}\left\{\frac{v^{\prime} e^{\nu}}{2 r}\right\}-\frac{\partial}{\partial r}\left\{\frac{1+e^{-\lambda}}{r^{2}}\right\}=0,
$$

which on using $e^{-\lambda}=y$ and $v^{\prime}=2 \bar{w}^{\prime} / \bar{w}$ can be rewritten as

$y^{\prime}+2\left(\frac{\bar{w}^{\prime \prime}-\frac{\bar{w}^{\prime}}{r}+\frac{\bar{w}}{r^{2}}}{\bar{w}^{\prime}-\frac{\bar{w}}{r}}\right) y+\frac{2 \bar{w}}{\left(\bar{w}^{\prime}-\frac{\bar{w}}{r}\right) r^{2}}=0$.

It is a first order differential equation in $y$. Its solution can be determined as

$y=e^{-\int \bar{f}_{1}(r) d r}\left(\int e^{-\int \bar{f}_{1}(r) d r} \bar{f}_{2}(r) d r+B\right)$,

where $B$ emerges as the integration constant and the values for $\bar{f}_{1}(r), \bar{f}_{2}(r)$ are given as

$$
\begin{aligned}
& \bar{f}_{1}(r)=2 \frac{d}{d r}\left[\ln \left(\bar{w}^{\prime}-\frac{\bar{w}}{r}\right)\right], \\
& \bar{f}_{2}(r)=\frac{-2 \bar{w}}{\left(\bar{w}^{\prime}-\frac{\bar{w}}{r}\right) r^{2}} .
\end{aligned}
$$

In terms of spacetime variables, we can rewrite the obtained solution as

$\frac{e^{\lambda / 2}}{r}\left(\gamma r^{2} e^{-v}-1\right)+\frac{1}{r}=\frac{v^{\prime}}{2}$, 
with $\gamma$ symbolizing the constant of integration. Utilization of junction conditions assists in the evaluation of $\gamma$ as

$\gamma=\frac{-4 M r_{\Sigma^{e}}+9 M^{2}}{r_{\Sigma^{e}}^{4}}$.

Usage of Eq. (39) generates the following outcome

$e^{v}=\gamma r^{2} \sin ^{2}\left(\int \frac{e^{\lambda / 2}}{r} d r+\sigma\right)$,

where $\sigma$ appears as integration constant. Upon using junction conditions, $\sigma$ is evaluated to be equal to

$\sigma=\sin ^{-1}\left[\sqrt{\frac{r_{\Sigma^{e}}^{2}\left(\frac{2 M}{r_{\Sigma^{e}}}-1\right)}{-4 M r_{\Sigma^{e}}+9 M^{2}}}\right]-\left(\int r^{-1} e^{\lambda / 2} d r\right)_{\Sigma^{e}}$.

So, the conformally flat condition resulted in the evaluation of only one generating function. To determine the complete solution, assumption of another constraint is requisite. For this reason, we consider vanishing radial pressure constraint i.e. $P_{r}=0$. Then Eq. (10) implies

$v^{\prime}=-\frac{1+e^{\lambda}}{r}-\frac{r e^{\lambda} \xi_{2}}{f_{R}}$.

Employing both the constraints $\left(P_{r}=0=\epsilon\right)$ in Eq. (18), we find

$\left(1+e^{\lambda}\right)^{2}+3 \lambda^{\prime} r+8\left(1+e^{\lambda}\right)-\lambda^{\prime} r e^{\lambda}-\eta_{1(D)}$,

which on assuming $2 g=e^{-\lambda}+1$ generates the following result

$g(9 g-4)-g^{\prime} r(3 g-2)-\frac{\eta_{2(D)}(2 g-1)^{2}}{4}=0$.

Integrating w.r.t radial coordinate, we determine the following relation

$\bar{C}_{1} r^{6}=\frac{4 g^{3}}{(9 g-4)}-6 r^{6} \int \frac{\eta_{2(D)} g^{2}(2 g-1)^{2}}{r^{7}(9 g-4)^{2}} d r$,

with $\bar{C}_{1}$ emerging as integration constant. Using Eqs. (39) and (41) together, we get

$e^{v}=\frac{\gamma r^{2} f_{R}^{2}(2 g-1)}{9 g^{2} f_{R}^{2}-4 g f_{R}^{2}+r^{4} \xi_{2}^{2}+6 g f_{R}^{2} r^{2} \xi_{2}}$.
The fluid variables turn out to be equal to

$$
\begin{aligned}
& |\mu|=\frac{f_{R}}{2 \pi}\left\{\frac{6 g^{2}-3 g+\eta_{2(D)}(2 g-1)^{2} / 8}{r^{2}(3 g-2)}\right\}+\xi_{1}, \\
& P_{\perp}=\frac{3 g^{2}}{4 \pi r^{2}(3 g-2)}+\frac{\eta_{2(D)} g(2 g-1)}{32 \pi r^{2}(3 g-2)}+\frac{\eta_{3(D)}}{8 \pi} .
\end{aligned}
$$

For $e^{v}$ to be positive, $g>2 / 3$ which coincides with the result of GR (Eq. (101) in [26]). This leads to a possibility that a thin shell emerges at the boundary $r=r_{\text {min }}$ in Palatini $f(R)$ gravity too. The generating functions $\Pi, z$ are determined as follows

$$
\begin{aligned}
& z(r)=\frac{2 f_{R}(g-1)-r^{2} \xi_{2}}{2 r f_{R}(2 g-1)}, \\
& \Pi(r)=-\frac{3 g^{2}}{4 \pi r^{2}(3 g-2)}-\frac{\eta_{2(D)} g(2 g-1)}{32 \pi r^{2}(3 g-2)}-\frac{\eta_{3(D)}}{8 \pi} .
\end{aligned}
$$

7.2 Solution obtained from zero complexity factor constraint

Just like GR, the scalar $Y_{T F}$ in Palatini $f(R)$ determines the complexity generated in the fluid configuration. So, it would be beneficial to look for a model for which the complexity factor vanishes i.e. $Y_{T F}=0$. Specifically, we need to assume an additional constraint besides this to obtain a realistic mathematical model. For this purpose, we take $P_{r}=0$. Thus

$v^{\prime}=\frac{-2 g}{r(2 g-1)}-\frac{r \xi_{2}}{f_{R}(2 g-1)}$.

The implementation of vanishing complexity factor leads to the following outcome

$$
\begin{aligned}
m_{T}= & \left(m_{T}\right)_{\Sigma_{e}}\left(\frac{r}{r_{\Sigma^{e}}}\right)^{3}+\left(\frac{\cosh \pi-1}{2}\right) r^{3} \\
& \times \int_{r}^{r_{\Sigma^{e}}} \frac{e^{(\nu+\lambda) / 2}}{\tilde{r}}\left(\frac{\xi_{1}-\xi_{2}-\xi_{3}}{2 f_{R}}-\chi_{1(D)}^{*}\right) d r .
\end{aligned}
$$

Using together the above two equations along with Eq. (24), the spacetime variable $e^{v}$ is found to be equal to

$$
\begin{aligned}
e^{v}= & \frac{1}{r^{2}(\cosh \pi-1)\left(2 g f_{R}+r^{2} \xi_{2}\right)} \\
& \times\left[4 r f _ { R } ( 2 g - 1 ) ^ { 1 / 2 } \left\{\left(m_{T}\right)_{\Sigma^{e}}\left(\frac{r}{r_{\Sigma^{e}}}\right)^{3}\right.\right. \\
& -\left(\frac{\cosh \pi-1}{2}\right) \\
\times & \left.r^{3} \int_{r}^{r_{\Sigma^{e}}} \frac{e^{(v+\lambda) / 2}}{\tilde{r}}\left(\frac{\xi_{1}-\xi_{2}-\xi_{3}}{2 f_{R}}+\chi_{1(D)}^{*}\right) d r\right\} \\
& \left.-\frac{\cosh \pi-1}{4} \int_{0}^{r} \tau_{(D)} d \tilde{r}\right]^{2} .
\end{aligned}
$$


The contribution of curvature amendments is clearly visible. For $f_{R}^{\prime}=0$ and $f(R)=R$, the result reduces to the findings of GR given by Eq. (129) in [26]. Employing $Y_{T F}=0$, we determine the following expression in terms of $g$

$g(5 g-2)+g^{\prime} r(1-g)-\eta_{3(D)} r^{2}(2 g-1)=0$.

Completing the derivative and integrating, we obtain

$$
\bar{C}_{2} r^{10}=\frac{g^{5}}{(5 g-2)^{3}}+10 r^{10} \int \frac{(2 g-1) \eta_{(3 D)} g^{4}}{r^{9}(5 g-2)^{5}} d r,
$$

with $\bar{C}_{2}$ appearing as integration constant. The fluid variables are determined as

$$
\begin{aligned}
& |\mu|=f_{R}\left[\frac{3 g(2 g-1)}{4 \pi r^{2}(g-1)}-\frac{\eta_{(3 D)}(2 g-1)}{4 \pi(g-1)}\right]-\frac{\xi_{1}}{8 \pi}, \\
& P_{\perp}=\frac{3 f_{R} g^{2}}{8 \pi r^{2}(g-1)}-\frac{g f_{R} \eta_{(3 D)}}{(g-1)}-\eta_{(4 D)},
\end{aligned}
$$

which clarifies that the fluid configuration in Palatini $f(R)$ gravitational theory is also restricted by the minimum value of the radial coordinate fulfilling the constraint $g\left(r_{\text {min }}\right)<1$ (because otherwise the fluid variable would become undefined). Such a minimum value i.e. $r_{\min }$ can be determined from Eq. (44). The existence of vacuum cavity, a thin shell and the negativity of Tolman mass follow in the same way as defined for GR [26] with a significant difference that $r_{\text {min }}$ determined from Eq. (44) involves the contribution of dark source elements too. This model possesses the generating functions defined as follows

$$
\begin{aligned}
z & =\frac{2 g f_{R}-2 f_{R}-r^{2} \xi_{2}}{2 r f_{R}(2 g-1)}, \\
\Pi & =-\frac{3 f_{R} g^{2}}{8 \pi r^{2}(g-1)}+\frac{g f_{R} \eta_{(3 D)}}{(g-1)}+\eta_{(4 D)} .
\end{aligned}
$$

It is evident that the curvature amendments affect the generating functions of the model too.

\subsection{Solution obtained from stiff equation of state}

To determine a mathematical model representing a realistic astronomical object, a thermodynamical equation called the equation of state $(\mathrm{EoS})$ is used. One of its types i.e. the stiff EoS is used for the fluid that is harder to compress and requires more support against the gravitational force e.g. Neutron stars. We utilized it here because it may provide of the possible solutions to Palatini field equations as it did for GR. The constraint that is followed by such EoS is the equivalence of energy density and radial pressure i.e. $|\mu|=P_{r}$. Hydrostatic equilibrium condition under this constraint takes the following form

$P_{r}^{\prime}+\frac{2 \Pi}{r}+\tilde{\chi}_{(D)}=0$.

To determine the generating functions, we need another constraint except $|\mu|=P_{r}$ because it is insufficient to obtain a solution to highly non-linear modified field equations. This calls for the assumption of two additional constraints mentioned below.

- Vanishing tangential pressure i.e. $P_{\perp}=0$ Considering $P_{\perp}=0$, the value of $P_{r}$ is determined by integrating Eq. (45) and producing

$|\mu|=P_{r}=\frac{\tilde{K}}{r^{2}} e^{\int \frac{x_{(D)}}{P_{r}} d r}$,

with $\tilde{K}$ expressing the integration constant. This expression demonstrates that the energy density as well as the radial pressure are influenced by the higher order curvature invariants of the theory. The mass function and the spacetime variables under these circumstances turn out to be as follows

$$
\begin{aligned}
m & =4 \pi \int_{0}^{r}\left(\frac{\tilde{K}}{f_{R}} e^{\int \frac{\chi_{(D)}}{P_{r}} d r}+\frac{\xi_{1} r^{2}}{8 \pi f_{R}}\right) d r, \\
e^{-\lambda} & =\frac{8 \pi}{r} \int_{0}^{r}\left(\frac{\tilde{K}}{f_{R}} e^{\int \frac{\chi_{(D)}}{P_{r}} d r}+\frac{\xi_{1} r^{2}}{8 \pi f_{R}}\right) d r-1, \\
\frac{v^{\prime}}{2} & =\frac{8 \pi \tilde{K} r e^{\int \frac{\chi_{(D)}}{P_{r}} d r}-\xi_{2} r^{3}-8 \pi f_{R} \int_{0}^{r}\left(\frac{\tilde{K}}{f_{R}} e^{\int \frac{\chi_{(D)}}{P_{r}} d r}+\frac{\xi_{1} r^{2}}{8 \pi f_{R}}\right) d r}{2 r f_{R}\left\{8 \pi \int_{0}^{r}\left(\frac{\tilde{K}}{f_{R}} e^{\int \frac{\chi_{(D)}}{P_{r}} d r}+\frac{\xi_{1} r^{2}}{8 \pi f_{R}}\right) d r-r\right\}} .
\end{aligned}
$$

In GR, the spacetime variable $v$ for such a model came out to be constant which represented the vanishing of the active gravitational mass. Here, we found that $v$ is not constant rather it is a function dependent on the dark source elements. This depicts that for the same mathematical model (obtained by using the constraints $|\mu|=P_{r}$ and $\left.P_{\perp}=0\right)$ under the influence of Palatini $f(R)$ gravity, the strength of the gravitational field generated by the astronomical body is non-zero and depends on the force exerted by the dark source constituents only.

So, for Palatini $f(R)$ gravity, the active gravitational mass is non-zero and the passive gravitational mass density i.e. $P_{r}-|\mu|$ vanishes identically since we are working with the constraint $P_{r}=|\mu|$. The generating functions for such a model are determined as

$$
z=\frac{8 \pi \tilde{K} r e^{\int \frac{\chi_{(D)}}{P_{r}} d r}-\xi_{2} r^{3}-8 \pi f_{R} \int_{0}^{r}\left(\frac{\tilde{K}}{f_{R}} \int \frac{\chi_{(D)}}{P_{r}} d r+\frac{\xi_{1} r^{2}}{8 \pi f_{R}}\right) d r}{2 r f_{R}\left\{8 \pi \int_{0}^{r}\left(\frac{\tilde{K}}{f_{R}} e^{\int \frac{\chi_{(D)}}{P_{r}} d r}+\frac{\xi_{1} r^{2}}{8 \pi f_{R}}\right) d r-r\right\}}+\frac{1}{r}
$$




$$
\Pi=\frac{\tilde{K}}{r^{2}} e^{\int \frac{\chi_{(D)}}{P_{r}} d r} .
$$

- Vanishing complexity factor i.e. $Y_{T F}=0$

When stiff equation of state and the vanishing complexity factor constraint are used simultaneously, the hydrostatic equilibrium condition yields

$P_{r}^{\prime \prime}+\frac{3 P_{r}^{\prime}}{r}+\omega_{(D)}=0$

with $\omega_{(D)}$ symbolizing the dark source elements. The solution to this second order differential equation is

$P_{r}=\frac{\tilde{b}}{r^{2}}-\tilde{a}-\omega_{(D)}^{*}$.

Clearly, the dark source constituents add to the stress acting in the radial direction. $\tilde{a}$ and $\tilde{b}$ correspond to positive constants of integration. The mass function for such a model turns out to be

$m=\frac{4 \pi r}{f_{R}}\left(b-\frac{a r^{2}}{3}\right)+\bar{\omega}_{(D)}$.

Using this equation and Eq. (13), one can easily determine the spacetime variable $\lambda$. The value for other spacetime variable $v$ can be found by substituting these results in Eq. (15). Further, we assume that the fluid configuration is bounded from the exterior region by the surface $\Sigma^{e}$ to obtain

$$
P_{r}=b\left[\frac{1}{r^{2}}-\frac{1}{r_{\Sigma^{e}}^{2}}\right]+\left(\omega_{(D)}^{*}\right)_{\Sigma^{e}}-\omega_{(D)}^{*} .
$$

The mass function becomes equal to

$$
m=\frac{4 \pi b r}{3 r_{\Sigma^{e}}^{2}}\left(3 r_{\Sigma^{e}}^{2}-r^{2}\right)+\tilde{\omega}_{(D)},
$$

whereas the Tolman mass becomes equal to

$$
\begin{aligned}
& \frac{4 \pi P_{r} r^{3}-\left(\xi_{1} / 2\right) r^{3}}{f_{R}}-m \\
& =\frac{-8 \pi b r^{3}}{3 r_{\Sigma e}^{2} f_{R}} \\
& \quad+\frac{4 \pi r^{3}}{f_{R}}\left\{\left(\omega_{(D)}^{*}\right)_{\Sigma^{e}}-\omega_{(D)}^{*}\right\}-\frac{\xi_{1}}{2 f_{R}}+\tilde{\omega}_{(D)} .
\end{aligned}
$$

The expression for $P_{\perp}$ reads

$$
\begin{aligned}
P_{\perp}= & \frac{r}{2}\left\{\left(\omega_{(D)}^{*}\right)_{\Sigma^{e}}-\omega_{(D)}^{*}\right\}^{\prime} \\
& -\frac{b}{r_{\Sigma e}^{2}}+\left(\omega_{(D)}^{*}\right)_{\Sigma^{e}}-\omega_{(D)}^{*}+\frac{r \chi(D)}{2} .
\end{aligned}
$$

It is evident that the active gravitational mass and the passive gravitational mass density are negative and zero respectively for this kind of model in Palatini $f(R)$ gravity.

\section{Discussion}

The Palatini $f(R)$ gravity is quite straightforward and uncomplicated generalization of GR, because it renders GR without requiring any kind of link between the metric and the connection. When tested via solar system tests, its results were found to be consistent with the observations. The fascinating mysteries of the universe like blackholes, gravitational waves and wormholes also exist in this theory [3436]. It incorporate no additional degrees of freedom instead, the dynamical equations are changed by the addition of new terms indicating the curvature amendments of the theory. Using a specific type of model, such curvature amendments provide an aid to comprehend the dark matter or dark energy epoch of the universe. The physical and mathematical models are modified by the curvature invariants involved in the theory to describe the expansionary phase of universe. One of the significant advantages of using Palatini $f(R)$ gravitational theory is that it works well also for the models that show certain inconsistencies when utilized with the metric version of $f(R)$ theory. An example is the cosmic speed-up problem modeled by $f(R)=R-\mu^{4} / R$ which when used with metric $f(R)$ gravity exhibits certain deviations from the observations, but when used with Palatini version, generates coherent results.

We have conducted a comprehensive study on the characteristics of hyperbolically symmetric fluid configurations with anisotropic stresses. The findings are parallel to the case of GR [26] where the manifold represents a 2-surface sphere from the exterior and hyperboloid of two sheets from the interior 2-surface. It is found that the fluid particles cannot reach the central region which results in the formation of a vacuole in that region. Negative energy density is requisite for the mass function to be positive in Palatini $f(R)$ gravity too. The contribution of dark source constituents can easily be seen in the expressions of the Weyl scalar and the mass function which demonstrates that the presence of dark source influence the tidal forces as well as the mass of hyperbolically symmetric astronomical object. The Tolman mass exhibits that in addition to the baryonic matter, the fluid is composed of dark source elements also. Few feasible solutions to such a type of symmetry in the manifold are then worked out, each one of which is defined by a different set of constraints to deal with the non-linearity of the modified gravitational equations. The obtained models resemble GR models with a considerable difference that their dynamical equations exhibit a physical behavior influenced by the higher order curva- 
ture invariants of the theory. All the outcomes are consistent with the findings of GR under the restriction $f(R)=R$ and $f_{R}^{\prime}=0$.

Acknowledgements The work of M. Z. Bhatti (PI) has been supported by National Research Project for Universities (NRPU), Higher Education Commission, Pakistan under research project No. 8769/Pun$\mathrm{jab} / \mathrm{NRPU} / \mathrm{R} \& \mathrm{D} / \mathrm{HEC} / 2017$. Also, the authors would like to thank the Higher Education Commission, Islamabad, Pakistan for its financial support through the Indigenous Ph.D. Fellowship For 5000 Scholars, Phase-II, Batch-V.

Data Availability Statement This manuscript has no associated data or the data will not be deposited. [Authors' comment: This manuscript is a mathematical framework, no graphical illustration is provided in it.]

Open Access This article is licensed under a Creative Commons Attribution 4.0 International License, which permits use, sharing, adaptation, distribution and reproduction in any medium or format, as long as you give appropriate credit to the original author(s) and the source, provide a link to the Creative Commons licence, and indicate if changes were made. The images or other third party material in this article are included in the article's Creative Commons licence, unless indicated otherwise in a credit line to the material. If material is not included in the article's Creative Commons licence and your intended use is not permitted by statutory regulation or exceeds the permitted use, you will need to obtain permission directly from the copyright holder. To view a copy of this licence, visit http://creativecomm ons.org/licenses/by/4.0/.

Funded by SCOAP ${ }^{3}$.

\section{Appendix}

The values for the factors $\xi_{1}, \xi_{2}$ and $\xi_{3}$ are

$$
\begin{aligned}
\xi_{1}= & \frac{\lambda^{\prime} e^{-\lambda} f_{R}^{\prime}}{2}-\frac{2 e^{-\lambda} f_{R}^{\prime}}{r}-e^{-\lambda} f_{R}^{\prime \prime} \\
& -\frac{3 e^{-\lambda} f_{R}^{\prime 2}}{2 f_{R}}+\frac{f_{R}}{2}\left(R-\frac{f}{f_{R}}\right), \\
\xi_{2}= & \frac{9 e^{-\lambda} f_{R}^{\prime 2}}{4 f_{R}}-\frac{v^{\prime} e^{-\lambda} f_{R}^{\prime}}{2} \\
& -\frac{2 e^{-\lambda} f_{R}^{\prime}}{r}+\frac{f_{R}}{2}\left(R-\frac{f}{f_{R}}\right), \\
\xi_{3}= & \frac{\lambda^{\prime} e^{-\lambda} f_{R}^{\prime}}{2}-\frac{f_{R}^{\prime} e^{-\lambda}}{r}+\frac{f_{R}^{\prime 2} e^{-\lambda}}{4 f_{R}} \\
& -\frac{v^{\prime} e^{-\lambda} f_{R}^{\prime}}{2}-e^{-\lambda} f_{R}^{\prime \prime}+\frac{f_{R}}{2}\left(R-\frac{f}{f_{R}}\right) .
\end{aligned}
$$

The value for the factor $\tilde{\chi}_{(D)}$ appearing in conservation equation has the following value

$$
\begin{aligned}
\tilde{\chi}(D)= & \frac{v^{\prime} \lambda^{\prime} e^{-\lambda} f_{R}^{\prime}}{32 \pi}-\frac{3 v^{\prime} e^{-\lambda} f_{R}^{\prime 2}}{32 \pi f_{R}}+\frac{\mu f_{R}^{\prime}}{2 f_{R}}-\frac{\lambda^{\prime} e^{-\lambda} f_{R}^{\prime 2}}{4 \pi f_{R}} \\
& +\frac{3 e^{-\lambda} f_{R}^{\prime 3}}{32 \pi f_{R}}-\frac{v^{\prime 2} e^{-\lambda} f_{R}^{\prime}}{32 \pi}-\frac{P_{r} f_{R}^{\prime}}{2 f_{R}}
\end{aligned}
$$

$$
\begin{aligned}
& -\frac{f_{R}^{\prime}}{f_{R}^{2}}\left(P_{r}-\frac{9 e^{-\lambda} f_{R}^{\prime 2}}{32 \pi f_{R}}-\frac{\nu^{\prime} e^{-\lambda} f_{R}^{\prime}}{16 \pi}-\frac{e^{-\lambda} f_{R}^{\prime}}{4 \pi r}\right) \\
& -\frac{\nu^{\prime \prime} e^{-\lambda} f_{R}^{\prime}}{16 \pi}+\frac{\lambda^{\prime} e^{-\lambda} f_{R}^{\prime}}{8 \pi r}-\frac{5 e^{-\lambda} f_{R}^{\prime}}{8 \pi r^{2}} \\
& +\frac{f_{R}^{\prime}}{8 \pi}\left(R-\frac{f}{f_{R}}\right)+f_{R}\left(R^{\prime}-\frac{f^{\prime}}{f_{R}}-\frac{f f_{R}^{\prime}}{f_{R}^{2}}\right)-\frac{f_{R}^{\prime} e^{-\lambda}}{8 \pi r^{2}} \\
& -\frac{f_{R}^{\prime 2} e^{-\lambda}}{2 \pi r f_{R}}-\frac{f_{R}^{\prime} P_{\perp}}{2 f_{R}}-\frac{f_{R}^{\prime}}{16 \pi f_{R}} \\
& \times\left(R-\frac{f}{f_{R}}\right)-\frac{P_{r} f_{R}^{\prime}}{f_{R}}-\frac{f_{R}}{16 \pi r}\left(R-\frac{f}{f_{R}}\right) \\
& -\frac{P_{\perp} f_{R}^{\prime}}{2 f_{R}}+\frac{f_{R}^{\prime} f_{R}^{\prime \prime} e^{-\lambda}}{16 \pi f_{R}} .
\end{aligned}
$$

The factors $\chi_{\delta \zeta}$ and $\psi_{\delta \zeta}$ appearing the expressions (27) and (28) have the following values

$$
\begin{aligned}
\chi_{\delta \zeta}= & \frac{3}{2 f_{R}}\left[\left(\nabla_{\alpha} f_{R} \nabla_{\delta} f_{R}\right) u_{\beta} u^{\delta}+\left(\nabla^{\theta} f_{R} \nabla_{\beta} f_{R}\right) u_{\alpha} u_{\theta}\right. \\
& \left.-\nabla_{\alpha} f_{R} \nabla_{\beta} f_{R}-\left(\nabla^{\theta} f_{R} \nabla_{\delta} f_{R}\right) u_{\theta} u^{\delta} g_{\alpha \beta}\right] \\
& \times \frac{1}{2}\left[\left(\nabla^{\theta} \nabla_{\theta} f_{R}\right) u_{\theta} u^{\delta} g_{\alpha \beta}+\nabla_{\alpha} \nabla_{\beta} f_{R}\right. \\
& \left.-\left(\nabla_{\alpha} \nabla_{\delta} f_{R}\right) u_{\beta} u^{\delta}-\left(\nabla^{\theta} \nabla_{\beta} f_{R}\right) u_{\alpha} u_{\theta}\right]-\frac{f-R f_{R}}{6} h_{\alpha \beta} \\
& -\frac{\left(\nabla f_{R}\right)^{2} h_{\alpha \beta}}{4 f_{R}}+\frac{\nabla^{\beta} f_{R} \nabla_{\beta} f_{R}}{2 f_{R}} h_{\alpha \beta}, \\
\psi_{\delta \zeta}= & \frac{\epsilon^{\epsilon} g_{a \alpha}}{8}\left[\epsilon_{\beta \pi \rho}\left\{\nabla^{\pi} \nabla_{\epsilon} f_{R}-\frac{3}{2 f_{R}}\left(\nabla^{\pi} f_{R} \nabla_{\epsilon} f_{R}\right)\right\}\right. \\
& +\epsilon_{\beta \pi \epsilon}\left\{\frac{3}{2 f_{R}}\left(\nabla^{\pi} f_{R} \nabla_{\rho} f_{R}\right)-\nabla^{\pi} \nabla_{\rho} f_{R}\right\} \\
& +\epsilon_{\theta \beta \rho}\left\{\frac{3}{2 f_{R}}\left(\nabla^{\theta} f_{R} \nabla_{\epsilon} f_{R}\right)-\nabla^{\theta} \nabla_{\epsilon} f_{R}\right\} \\
& \left.+\epsilon_{\theta \beta \epsilon}\left\{\nabla^{\theta} \nabla_{\rho} f_{R}-\frac{3}{2 f_{R}}\left(\nabla^{\theta} f_{R} \nabla_{\rho} f_{R}\right)\right\}\right] \\
& -\frac{f-R f_{R}}{6} h_{\alpha \beta}-\frac{\left(\nabla f_{R}\right)^{2} h_{\alpha \beta}}{2 f_{R}}+\frac{\nabla^{\gamma} f_{R} \nabla_{\gamma} f_{R}}{2 f_{R}} h_{\alpha \beta} .
\end{aligned}
$$

The factors $\bar{\chi}_{(D)}$ and $\bar{\psi}_{(D)}$ have the following values

$$
\begin{aligned}
\bar{\psi}_{(D)}= & \frac{3\left(\nabla f_{R}\right)^{2}}{2 f_{R}} \\
& +\frac{1}{4}\left[\left(\frac{3}{2 f_{R}} \nabla^{\pi} f_{R} \nabla_{\epsilon} f_{R}-\nabla^{\pi} \nabla_{\epsilon} f_{R}\right) h_{\pi}^{\epsilon}\right. \\
& +\left(\frac{3}{2 f_{R}} \nabla^{\pi} f_{R} \nabla_{\rho} f_{R}-\nabla^{\pi} \nabla_{\rho} f_{R}\right) h_{\pi}^{\rho} \\
& +\left(\frac{3}{2 f_{R}} \nabla^{\theta} f_{R} \nabla_{\epsilon} f_{R}-\nabla^{\theta} \nabla_{\epsilon} f_{R}\right) h_{\theta}^{\epsilon} \\
& \left.+\left(\frac{3}{2 f_{R}} \nabla^{\theta} f_{R} \nabla_{\rho} f_{R}-\nabla^{\theta} \nabla_{\rho} f_{R}\right) h_{\theta}^{\rho}\right] \\
& -\frac{3 \nabla^{\gamma} f_{R} \nabla_{\gamma} f_{R}}{2 f_{R}}+\left(\frac{f-R f_{R}}{2}\right), \\
\bar{\chi}(D)= & \left(\frac{3}{2 f_{R}} \nabla^{\alpha} f_{R} \nabla_{\rho} f_{R}-\frac{\nabla^{\alpha} \nabla_{\delta} f_{R}}{2}\right) u_{\alpha} u^{\delta}
\end{aligned}
$$




$$
\begin{aligned}
& +\left(\frac{3}{2 f_{R}} \nabla^{\theta} \nabla_{\alpha} f_{R}-\frac{\nabla^{\theta} \nabla_{\alpha} f_{R}}{2}\right) u^{\alpha} u_{\theta} \\
& +2\left(\nabla^{\theta} \nabla_{\delta} f_{R}-\frac{3 \nabla^{\theta} f_{R} \nabla_{\delta} f_{R}}{f_{R}}\right) u_{\theta} u^{\rho} \\
& -\left(\frac{f-R f_{R}}{2}\right)-\frac{3 \square f_{R}}{2 f_{R}}+\frac{3 \nabla^{\gamma} f_{R} \nabla_{\gamma} f_{R}}{2 f_{R}} \\
& +\frac{\square f_{R}}{2}-\frac{3\left(\nabla f_{R}\right)^{2}}{4 f_{R}} .
\end{aligned}
$$

The factors $\eta_{1(D)}, \eta_{2(D)}, \eta_{3(D)}$ and $\eta_{4(D)}$ have the following values

$$
\begin{aligned}
\eta_{1(D)=} & 8 r^{2} e^{\lambda}\left(-e^{-\lambda}\left(\frac{r e^{\lambda} \xi_{2}}{4 f_{R}}\right)^{\prime}-\frac{r^{2} \xi_{2}^{2}}{32 f_{R}^{2}}-\frac{\lambda^{\prime} r \xi_{2}}{8 f_{R}}-\frac{\xi_{2}}{2 f_{R}}\right), \\
\eta_{2(D)=} & -(2 g-1)\left(\frac{r \xi_{2}}{4 f_{R}(2 g-1)}\right)^{\prime}-\frac{r^{2} \xi_{2}^{2}}{32 f_{R}^{2}} \\
& -\frac{\xi_{2}}{4 f_{R}(2 g-1)}+\frac{2 r \xi_{2} g^{\prime}}{8 f_{R}(2 g-1)}-\frac{\xi_{2}}{2 f_{R}}, \\
\eta_{3(D)=}= & \frac{r^{2} \xi_{2}^{2}+2 f_{R} g \xi_{2}}{4 f_{R}^{2}(2 g-1)} \\
& -\frac{9 g^{2} r \xi_{2}-4 r g \xi_{2} \eta_{2(D)} r \xi_{2}(2 g-1)^{2} / 4}{2 r f_{R}(2 g-1)(3 g-2)} \\
& -\frac{2 g-1}{2}\left(\frac{r \xi_{2}}{f_{R}(2 g-1)}\right)^{\prime}+\xi_{3}, \\
\eta_{(4 D)}= & -\frac{f_{R}(2 g-1)}{2}\left(\frac{r \xi_{2}}{f_{R}(2 g-1)}\right)^{\prime} \\
& +\frac{r^{3} \xi_{2}^{2}+4 r f_{R} g \xi_{2}-2 r^{2} f_{R} g^{\prime} \xi_{2}-2 f_{R} r \xi_{2}(2 g-1)}{4 r f_{R}(2 g-1)}+\xi_{3} .
\end{aligned}
$$

The factor $\omega_{(D)}, \omega_{(D)}^{*}$ and $\bar{\omega}_{(D)}$ have the following values

$$
\begin{aligned}
\omega_{(D)}= & -\frac{P_{r}^{\prime} f_{R}^{\prime}}{f_{R}}-\frac{P_{r} f_{R}^{\prime}}{r f_{R}}+\frac{f_{R}}{r^{4}} \\
& \times\left\{\frac{r^{4} \chi_{(D)}}{f_{R}}+\frac{3 \xi_{1} r^{2}}{2 f_{R}}+\frac{r^{3}\left(\xi_{1}-\xi_{2}-\xi_{3}\right)}{8 \pi f_{R}}-\frac{f_{R} \chi_{(D)}^{*}}{8 \pi}\right\}^{\prime}, \\
\omega_{(D)}^{*}= & \int\left(\frac{1}{r^{3}} \int \omega_{(D)} r^{3} d r\right) d r, \\
\bar{\omega}_{(D)}= & \frac{4 \pi}{f_{R}} \int_{0}^{r} \omega_{(D)}^{*} r^{2} d r \\
& +\int_{0}^{r}\left\{-\frac{\xi_{2} r^{2}}{2 f_{R}}+\frac{f_{R}^{\prime}}{f_{R}^{2}}\left(b r-\frac{a r^{3}}{3}-\int_{0}^{r} \omega_{(D)}^{*} r^{2} d r\right)\right\} d r .
\end{aligned}
$$

\section{References}

1. L. Herrera, A. Di Prisco, J. Ospino, L. Witten, Phys. Rev. D 101, 064071 (2020)

2. L. Herrera, L. Witten, Adv. High Energy Phys. 2018, 3839103 (2018)

3. M.D. Kruskal, Phys. Rev. 119, 1743 (1960)

4. B.K. Harrison, Phys. Rev. 116, 1285 (1959)
5. M. Gaudin, V. Gorini, A. Kamenshchik, U. Moschella, V. Pasquier, Int. J. Mod. Phys. D 15, 1387 (2006)

6. A.Yu. Kamenshchik, E.O. Pozdeeva, A.A. Starobinsky, A. Tronconi, T. Vardanyan, G. Venturi, S.Yu. Verno, Phys. Rev. D 98, 124028 (2018)

7. A. Maciel, M. Le Delliou, J.P. Mimoso, Class. Quantum Gravity 37, 125005 (2020)

8. L. Rizzi, S.L. Cacciatori, V. Gorini, A. Kamenshchik, O.F. Piattella, Phys. Rev. D 82, 027301 (2010)

9. G. Ellis, J. Math. Phys. 8, 1171 (1967)

10. F.S.N. Lobo, J.P. Mimoso, Phys. Rev. D 82, 044034 (2010)

11. S. Nojiri, S.D. Odintsov, Int. J. Geom. Methods Mod. Phys. 4, 115 (2007)

12. X. Meng, P. Wang, Phys. Lett. B 584, 1 (2004)

13. S. Nojiri, S.D. Odintsov, Phys. Lett. B 576, 5 (2003)

14. O. Bertolami, C.G. Böhmer, T. Harko, F.S.N. Lobo, Phys. Rev. D 75, $104016(2007)$

15. B.C. Paul, P.S. Debnath, S. Ghose, Phys. Rev. D 79, 083534 (2009)

16. F.S.N. Lobo, M.A. Oliveira, Phys. Rev. D 80, 104012 (2009)

17. G.J. Olmo, D. Rubiera-Garcia, A. Wojnar, Phys. Rep. 876, 1 (2020)

18. T. Clifton, P.G. Ferreira, A. Padilla, C. Skordis, Phys. Rep. 513, 1 (2012)

19. L. Herrera, A. Di Prisco, J. Ospino, Symmetry 13, 1568 (2021)

20. C. Lanczos, Rev. Mod. Phys. 34, 379 (1962)

21. L. Herrera, A. Di Prisco, J. Ibáñez, J. Ospino, Phys. Rev. D 87, 024014 (2013)

22. M.Z. Bhatti, Z. Yousaf, Z. Tariq, Chin. J. Phys. 72, 18 (2021)

23. M.Z. Bhatti, Z. Yousaf, Z. Tariq, Phys. Scr. 96, 115301 (2021)

24. M.Z. Bhatti, Z. Tariq, Phys. Dark Universe 29, 100600 (2020)

25. M.Z. Bhatti, Z. Yousaf, Z. Tariq, Eur. Phys. J. Plus 136, 857 (2021)

26. L. Herrera, A. Di Prisco, J. Ospino, Phys. Rev. D 103, 024037 (2021)

27. Z. Tariq, M.Z. Bhatti, Z. Yousaf, Int. J. Mod. Phys. D 30, 2150058 (2021)

28. L. Herrera, A. Di Prisco, J. Ospino, Can. J. Phys. 96, 1010 (2018)

29. L. Herrera, A. Di Prisco, J. Ibáñez, J. Ospino, Phys. Rev. D 89, 084034 (2014)

30. L. Herrera, A. Di Prisco, J. Ospino, Eur. Phys. J. C 76, 603 (2016)

31. S. Capozziello, M. De Laurentis, V. Faraoni, Open Astron. J. 3, 49 (2010)

32. Z. Yuusaf, Phys. Scr. 95, 075307 (2020)

33. L. Herrera, J. Ospino, A. Di Prisco, E. Fuenmayor, O. Troconis, Phys. Rev. D 79, 064025 (2009)

34. A. Wojnar, Phys. Rev. D 102, 124045 (2020)

35. C. Barragan, G.J. Olmo, H. Sanchis-Alepuz, Phys. Rev. D 80, 024016 (2009)

36. M. Szyd-Lowski, A. Stachowski, A. Borowiec, Eur. Phys. J. C 77, 603 (2017) 